\title{
Role of ferritinophagy in cystine deprivation-induced cell death in glioblastoma cells
}

$\operatorname{AUTHOR}(\mathrm{S})$ :

Hayashima, Kazuki; Kimura, Ikuo; Katoh, Hironori

\section{CITATION:}

Hayashima, Kazuki ... [et al]. Role of ferritinophagy in cystine deprivation-induced cell death in glioblastoma cells. Biochemical and Biophysical Research Communications 2021, 539: 56-63

ISSUE DATE:

2021-02

URL:

http://hdl.handle.net/2433/267434

\section{RIGHT:}

(c) 2021. This manuscript version is made available under the Creative Commons Attribution-NonCommercialNoDerivatives 4.0 International license.; The full-text file will be made open to the public on 5 February 2022 in accordance with publisher's 'Terms and Conditions for Self-Archiving'.; This is not the published version. Please cite only the published version. この論文は出版社版でありません。引用の際には出版社版をご確認ご利用ください。 


\section{Role of ferritinophagy in cystine deprivation-induced cell death in glioblastoma cells}

Kazuki Hayashima, Ikuo Kimura, and Hironori Katoh

Laboratory of Molecular Neurobiology, Graduate School of Biostudies,

Kyoto University, Yoshidakonoe-cho, Sakyo-ku, Kyoto 606-8501, Japan

Address all correspondence to Hironori Katoh, Laboratory of Molecular

Neurobiology, Graduate School of Biostudies, Kyoto University,

Yoshidakonoe-cho, Sakyo-ku, Kyoto 606-8501, Japan

Tel: $+81-75-753-7687 ;$ Fax: $+81-75-753-7688$

E-mail address: hirokato@pharm.kyoto-u.ac.jp 


\section{Abstract}

Ferroptosis is a form of cell death caused by iron-dependent lipid peroxidation. Cancer cells increase cystine uptake for the synthesis of glutathione (GSH), which is used by glutathione peroxidase 4 to reduce lipid peroxides. Here, we report that cystine deprivation in glioblastoma cells, but not inhibition of GSH synthesis by L-buthionine sulfoximine (BSO), induces ferroptosis. We found that cystine deprivation decreased the protein levels of ferritin heavy chain FTH1, whereas it was increased by BSO treatment. The lysosome inhibitor bafilomycin A1 or deletion of nuclear receptor coactivator 4 (NCOA4) inhibited cystine deprivation-induced decrease in FTH1 protein levels and cell death. In addition, cystine deprivation induced microtubule-associated protein light chain 3 (LC3)-II protein accumulation, suggesting that cystine deprivation induces ferritinophagy. BSO causes cell death when glioblastoma cells are treated with iron inducers, ferrous ammonium sulfate or hemin. On the other hand, cystine deprivation-induced degradation of FTH1 and cell death required glutamine. This study suggests 
that ferritinophagy, in addition to GSH depletion, plays an important role in cystine deprivation-induced ferroptosis in glioblastoma cells.

\section{Keywords}

Cell death; glutathione; cystine; ferritin; glioblastoma.

\section{Abbreviations}

GSH, glutathione; BSO, L-buthionine sulfoximine; NCOA4, nuclear receptor coactivator 4; LC3, microtubule-associated protein light chain 3; GPX4, glutathione peroxidase 4; DFO, deferoxamine; BafA1, bafilomycin A1; sgRNA, single guide RNA; LDH, lactate dehydrogenase; ANOVA, analysis of variance 


\section{Introduction}

Glioblastoma is the most common malignant brain tumor with a poor prognosis $[1,2]$. Currently, patients with glioblastoma are usually treated by surgical excision, radiation, and chemotherapy, but no effective treatment has been established and the 5-year survival rate is only 5\%. Therefore, it is important to find novel therapeutic targets for effective treatment of glioblastoma.

Ferroptosis is a form of cell death that causes excessive irondependent oxidative damage to lipids [3]. It is well known that inhibition of glutathione peroxidase 4 (GPX4), an enzyme that directly reduces lipid peroxide in a glutathione (GSH)-dependent manner, induces ferroptosis [4]. The cystine/glutamate antiporter system $\mathrm{x}_{c}^{-}$is composed of two subunits, a catalytic light chain $\mathrm{xCT}$ (SLC7A11) and a regulatory heavy chain CD98 (4F2hc or SLC3A2), and exchanges extracellular cystine for intracellular glutamate at the plasma membrane $[5,6]$. In many cancer cells, the imported cystine is a major source of cysteine required for GSH synthesis and the 
activity of GPX4. Therefore, deprivation of cystine or treatment with pharmacological inhibitors of $\mathrm{xCT}$, such as erastin, causes ferroptosis in several types of cancer cells [7]. Thus, intracellular transport of cystine is important to avoid oxidative stress and cell death in cancer cells. On the other hand, xCT promotes cell death under glucose-limited conditions [8-10].

One of key characteristic features of ferroptosis is that cell death is inhibited by iron chelators such as deferoxamine (DFO). Several proteins involved in iron metabolism regulate ferroptosis $[11,12]$. Transferrin and transferrin receptor, which import iron into cells, are required for amino acid deprivation- or erastin-induced ferroptosis $[13,14]$. Ferritin is a major iron storage protein composed of heavy chain subunit (FTH1) and light chain subunit (FTL), and nuclear receptor coactivator 4 (NCOA4)-mediated lysosomal degradation of ferritin (ferritinophagy) increases the sensitivity to ferroptosis [15-18]. Knockdown of the iron exporter ferroportin (SLC40A1), or Prominin2, which promotes exosomal export of ferritin, also promotes ferroptosis $[19,20]$. On the other hand, inhibitors or knockdown of heme 
oxygenase-1, which catalyzes the degradation of heme to produce ferrous iron, suppresses ferroptosis $[21,22]$. However, it remains unclear how these iron supply systems are used properly in ferroptosis induced by different inducers. We demonstrate that cystine deprivation, but not GSH depletion, induces ferroptosis in glioblastoma cells. We found that regulation of both GSH and iron levels is required for cystine deprivation-induced ferroptosis.

\section{Materials and Methods}

\section{Reagents and antibodies}

BSO and DFO were purchased from Santa Cruz Biotechnology (BSO, sc-200824, $100 \mu \mathrm{M}$; DFO, sc-203331, $100 \mu \mathrm{M}$ ). Ferrostatin-1 (Fer1) was purchased from Sigma-Aldrich (SML0583, $1 \mu \mathrm{M}$ ). Bafilomycin A1 (BafA1) was from Cayman Chemical (No. 11038, $1 \mu \mathrm{M}$ ). Hemin was purchased from Fujifilm (No. 089-10321, $10 \mu \mathrm{M}$ ). We used the following antibodies in this study: rabbit monoclonal antibodies against FTH1 (D1D4, \#4393) and NCOA4 (E8H8Z,\#66849) (Cell Signaling Technology); a mouse 
monoclonal antibody against transferrin receptor (CD71, sc-65882) (Santa

Cruz Biotechnology); a rabbit polyclonal antibody against microtubuleassociated protein light chain 3 (LC3) (MBL, PM036); a mouse monoclonal antibody against $\alpha$-tubulin (Sigma-Aldrich, T5168); and secondary antibodies conjugated to horseradish peroxidase (DAKO)

\section{Cell culture and transfection}

T98G and A172 cells were provided by the RIKEN BRC through the National Bio-Resource Project of the MEXT, Japan (T98G, RCB1954; A172, RCB2530). They were grown in Dulbecco's modified Eagle's medium containing 10\% fetal bovine serum (FBS), $4 \mathrm{mM}$ glutamine, 100 units $/ \mathrm{ml}$ of penicillin, and $0.1 \mathrm{mg} / \mathrm{ml}$ of streptomycin under humidified air containing $5 \% \mathrm{CO}_{2}$ at $37^{\circ} \mathrm{C}$. We used the CRISPR/Cas9-mediated homology-independent knock-in system [23] to generate NCOA4-deficient T98G cells. The single guide RNA (sgRNA) targeting NCOA4 sequence (5'ggtatggctgtatgaacagg-3') was designed based on a previous report [24] and 
cloned into the sgRNA expression vector peSpCAS9(1.1)-2xsgRNA (Addgene plasmid \#80768). T98G cells were seeded in two 60-mm dishes $\left(2.5 \times 10^{5}\right.$ cells/dish $)$ and co-transfected with peSpCAS9(1.1)-2xsgRNA containing sgRNA targeting NCOA4 and pDonor-tBFP-NLS-Neo (Addgene plasmid \#80766) using Lipofectamine 2000 (Life Technologies). Two days after transfection, the cells were placed in the medium containing $250 \mu \mathrm{g} / \mathrm{ml}$ G418 (Wako) to eliminate untransfected cells. Ten days after selection, colonies grown from single cells were isolated, and they were expanded and screened by immunoblotting with anti-NCOA4 antibody. Control T98G cells were obtained by transfection with peSpCAS9(1.1)-2xsgRNA and pDonortBFP-NLS-Neo.

\section{Cell death experiment}

The medium without cystine was prepared by dissolving $0.2 \mathrm{~g}$ of $\mathrm{CaCl}_{2}, 0.1 \mathrm{mg}$ of $\mathrm{Fe}\left(\mathrm{NO}_{3}\right)_{3} / 9 \mathrm{H}_{2} \mathrm{O}, 97.67 \mathrm{mg}$ of $\mathrm{MgSO}_{4}, 0.4 \mathrm{~g}$ of $\mathrm{KCl}, 3.7 \mathrm{~g}$ of $\mathrm{NaHCO}_{3}, 6 \mathrm{~g}$ of $\mathrm{NaCl}, 0.109 \mathrm{~g}$ of $\mathrm{NaH}_{2} \mathrm{PO}_{4}$, and $40 \mathrm{ml}$ of minimum 
essential medium vitamin solution $(100 \times)$ liquid (Thermo Fisher Scientific) in 11 of water with the following concentrations of amino acids: arginine$\mathrm{HCl}(0.4 \mathrm{mM})$, histidine- $\mathrm{HCl}-\mathrm{H}_{2} \mathrm{O}(0.2 \mathrm{mM})$, isoleucine $(0.8 \mathrm{mM})$, leucine (0.8 mM), lysine- $\mathrm{HCl}(0.8 \mathrm{mM})$, methionine $(0.2 \mathrm{mM})$, phenylalanine $(0.4$ $\mathrm{mM})$, threonine $(0.8 \mathrm{mM})$, tryptophan $(0.08 \mathrm{mM})$, tyrosine-2Na-2 $\mathrm{H}_{2} \mathrm{O}(0.4$ $\mathrm{mM})$, valine $(0.8 \mathrm{mM})$, glutamine $(2 \mathrm{mM})$. For cystine deprivation, cells were plated in a 48-well plate (Greiner Bio-One, 677180) and cultured for $24 \mathrm{~h}$. Then they were rinsed twice with phosphate-buffered saline (PBS), and the medium was replaced with cystine-free medium containing $10 \%$ dialyzed FBS (HyClone) with or without $0.2 \mathrm{mM}$ cystine for $24 \mathrm{~h}$. Cell death was measured by lactate dehydrogenase (LDH) release assay using the MTX LDH kit (Kyokuto Pharmaceutical Industrial) according to the manufacturer's instructions. The optical density was measured at $595 \mathrm{~nm}$ using a microplate reader (Tecan, GENious). The value of LDH release after treatment with $0.1 \%$ Tween 20 was defined as $100 \%$ cell death. 


\section{Measurement of cellular GSH}

Cells were seeded onto 96-well plates (Greiner Bio-One, 655180,

1,000 cells/well) and cultured for $24 \mathrm{~h}$. Then they were rinsed twice with

PBS, and the medium was replaced with cystine-free medium containing $10 \%$ dialyzed FBS with or without $0.2 \mathrm{mM}$ cystine or with $100 \mu \mathrm{M}$ BSO for

15 h. Cellular GSH level was measured using the GSH-Glo Glutathione Assay (Promega). Luminescence was measured using the microplate reader.

\section{Immunoblotting}

Cells were seeded in 24-well plates (Greiner Bio-One, 662160, $4 \times$ $10^{4}$ cells/well) and cultured for $24 \mathrm{~h}$. Then they were rinsed twice with PBS, and the medium was replaced with cystine-free medium with or without 0.2 $\mathrm{mM}$ cystine for the indicated times. Cells were washed with PBS and lysed with Laemmli sample buffer. The proteins in the cell lysates were separated by SDS-PAGE and transferred onto a polyvinylidene difluoride membrane (Millipore Corporation). The membrane was blocked with 3\% low fat milk 
in Tris-buffered saline, and then incubated with primary antibodies diluted with $1 \%$ low fat milk or Can Get Signal (TOYOBO). The primary antibodies were detected with horseradish peroxidase-conjugated secondary antibodies and ECL western blotting detection reagents (GE Healthcare Life Sciences). The signals were captured by Amersham Imager 600 (GE Healthcare Life Sciences).

\section{Data analysis}

Data were analyzed using analysis of variance (ANOVA) with Tukey's honestly significant difference post hoc test. $p<0.05$ was considered significant. Statistical analyses were performed using KaleidaGraph (Synergy Software).

\section{Results}

Cystine deprivation, but not glutathione depletion, induces ferroptosis in glioblastoma cells. 
It is well known that inactivation of GPX4 by the GPX4 inhibitor RSL3 or indirect suppression of GSH synthesis (treatment with L-buthionine sulfoximine (BSO), an inhibitor of $\gamma$-glutamylcysteine synthetase, or cystine deprivation) can induce ferroptosis $[3,4,7,11]$. We used two glioblastoma cell lines, T98G and A172, to explore the mechanisms underlying cystine deprivation-induced ferroptosis. We measured LDH release in the culture medium $24 \mathrm{~h}$ after cystine deprivation, and found that cystine deprivation induced cell death in both cell lines. However, BSO had little effect on cell viability (Fig. 1A). We confirmed that the levels of intracellular GSH decreased after cystine deprivation or treatment with BSO (Fig. 1B). The selective ferroptosis inhibitor Fer-1 and the iron chelator DFO suppressed cystine deprivation-induced cell death (Fig. 1C). This suggests that inhibition of GSH synthesis is not sufficient to induce ferroptosis in glioblastoma cells.

We next examined the effect of cystine deprivation or BSO treatment on the expression levels of proteins involved in iron metabolism. 
Cystine deprivation decreased the protein level of ferritin heavy chain FTH1 in both T98G and A172 cells, whereas the level of transferrin receptor CD71 did not change after cystine deprivation (Fig. 1D). On the other hand, the levels of FTH1 increased when the cells were treated with BSO (Fig. 1E). Thus, this suggest that regulation of FTH1 levels differs between cystine deprivation and BSO treatment in glioblastoma cells.

Ferritinophagy plays an important role in cystine deprivation-induced cell death.

Previous studies reported that FTH1 is degraded in lysosomes in response to several ferroptosis inducers [15-18]. The lysosome inhibitor bafilomycin A1 (BafA1) increased the levels of FTH1 protein under cystine deprivation in T98G and A172 cells (Fig. 2A), suggesting that FTH1 is degraded in lysosomes under cystine deprivation. BafA1 also suppressed cystine deprivation-induced cell death in T98G and A172 cells (Fig. 2B).

Ferritinophagy is the autophagic degradation of ferritin, and 
NCOA4 is required for the delivery of ferritin to lysosomes and ferritinophagy [25-27]. To examine whether NCOA4 is involved in the cystine deprivation-induced cell death of glioblastoma cells, we generated NCOA4-deficient T98G cells using CRISPR/Cas9-mediated deletion of NCOA4 (Fig. 2C). Cystine deprivation decreased the protein level of FTH1 in control sgRNA-expressing T98G cells (Control KO), whereas it had little effect on the level of FTH1 in NCOA4-deficient cells (NCOA4 KO) (Fig. 2D). In addition, cystine deprivation did not induce cell death in NCOA4deficient cells (Fig. 2E). There is a close relationship between the amount of microtubule-associated protein light chain 3 (LC3)-II and autophagosome formation [28, 29]. To confirm that cystine deprivation induces autophagosome formation, T98G cell lysates were analyzed by immunoblotting with anti-LC3 antibody, and cystine deprivation increased the amount of LC3-II, which was further increased by treatment with BafA1 (Fig. 2F). These results suggest that cystine deprivation induces NCOA4mediated ferritinophagy to induce ferroptosis in glioblastoma cells. 


\section{Intracellular iron accumulation induces cell death in BSO-treated cells.}

When ferrous ammonium sulfate (FAS) or hemin is added into the medium, the level of intracellular iron increases [30]. Therefore, we next examined the effects of FAS and hemin on cell viability in glioblastoma cells. Treatment with FAS alone had little effect on the viability of T98G and A172 cells. However, treatment with FAS and BSO significantly increased cell death in both cell lines (Fig. 3A). Hemin also increased the death of BSOtreated cells but not in untreated cells (Fig. 3B). This suggests that GSH depletion causes cell death when accompanied by intracellular iron accumulation in glioblastoma cells. We also found that treatment with FAS or hemin restored cystine deprivation-induced cell death in T98G and A172 cells in the presence of BafA1 (Fig. 3C and 3D). This suggests that lysosomal activity plays a role in the iron accumulation required for cystine deprivation-induced cell death. 
Glutamine is required for cystine deprivation-induced degradation of

\section{FTH1 and cell death.}

Previous studies reported that glutamine is required for cystine/cysteine deprivation-induced ferroptosis $[14,31]$. Consistent with this, cystine deprivation failed to induce cell death in the absence of glutamine in T98G and A172 cells (Fig. 4A). Treatment of the cells with FAS partly restored cystine deprivation-induced cell death in the absence of glutamine (Fig. 4B). In addition, glutamine deprivation suppressed the cystine deprivation-induced decrease in the protein level of FTH1 (Fig. 4C), suggesting that glutamine is required for ferritinophagy in response to cystine deprivation in glioblastoma cells. On the other hand, glutamine deprivation alone reduced the intracellular GSH levels, and deprivation of cystine alone and deprivation of cystine with glutamine induced GSH depletion at similar levels (Fig. 4D), suggesting that glutamine is dispensable for cystine deprivation-induced GSH depletion. 


\section{Discussion}

Ferroptosis is a form of cell death caused by lipid peroxidation in an iron-dependent manner. It is well known that cystine deprivation or $\mathrm{xCT}$ inhibitors induce GSH depletion, which results in inactivation of GPX4, leading to lipid peroxidation and cell death. We demonstrated that in glioblastoma cells, cystine deprivation, but not BSO treatment, causes ferroptosis, although they both cause cellular GSH depletion. NCOA4mediated degradation of FTH1 was observed in cystine-deprived cells but not in BSO-treated cells. The inhibition of FTH1 degradation by BafA1 or NCOA4 deletion suppressed cystine deprivation-induced cell death. In addition, cell death was noted when BSO treatment accompanied iron supplementation. This suggests that cystine deprivation-induced ferroptosis requires not only GSH depletion, but also intracellular iron accumulation. On the other hand, we also found that glutamine is required for cystine deprivation-induced FTH1 degradation.

The major function of ferritin is to store excess iron, and the release 
of iron from ferritin requires its lysosomal degradation $[32,33]$. NCOA4 delivers ferritin to lysosomes for degradation [25]. Ferritinophagy is a form of autophagy involving NCOA4-mediated degradation of ferritin [26, 27], and recent studies reported that ferritinophagy promotes ferroptosis [15-18]. In glioblastoma cells, cystine deprivation, but not BSO treatment, induces ferritinophagy and ferroptosis. On the other hand, BSO treatment induces cell death when cells are simultaneously treated with iron inducers. These results suggest that ferritinophagy plays an essential role in cystine deprivation-induced ferroptosis, and that cystine deprivation-induced ferritinophagy is not downstream of GSH depletion in glioblastoma cells. Thus, it is important to elucidate the mechanism by which cystine deprivation induces ferritinophagy in glioblastoma cells.

In the absence of glutamine, cystine deprivation fails to induce ferritin degradation, suggesting that glutamine is required for the lysosomal degradation of ferritin in response to cystine deprivation. As FAS treatment does not fully restore cystine deprivation-induced cell death without 
glutamine, we cannot exclude the possibility that glutamine is required for steps other than intracellular iron accumulation during ferroptosis induced by cystine deprivation. Glutamine is metabolized to glutamate and subsequently to $\alpha$-ketoglutarate used in the tricarboxylic acid cycle, which has been reported to play a crucial role in cysteine-deprivation-induced ferroptosis $[14,31]$. Further studies are required to clarify how glutamine is used to induce degradation of ferritin in response to cystine deprivation.

\section{Acknowledgments}

We thank Dr. Yohei Katoh and Prof. Kazuhisa Nakayama (Kyoto University) for providing peSpCAS9(1.1)-2xsgRNA and pDonor-tBFPNLS-Neo. This study was supported in part by Grants-in-aid for Scientific Research from the Japan Society for the Promotion of Science (18K06215) and Princess Takamatsu Cancer Research Fund Grant (18-25006).

\section{References}


[1] T.A. Dolecek, J.M. Propp, N.E. Stroup, C. Kruchko, CBTRUS statistical report: primary brain and central nervous system tumors diagnosed in the United States in 2005-2009, Neuro Oncol. 14 (2012) v1-49.

[2] S.K. Tan, A. Jermakowicz, A.K. Mookhtiar, C.B. Nemeroff, S.C. Schürer, N.G. Ayad, Drug repositioning in glioblastoma: a pathway perspective, Front. Pharmacol.16 (2018) 218.

[3] S.J. Dixon, K.M. Lemberg, M.R. Lamprecht, R. Skouta, E.M. Zaitsev, C.E. Gleason, D.N. Patel, A.J. Bauer, A.M. Cantley, W.S. Yang, B. Morrison III, B.R. Stockwell, B.R. (2012) Ferroptosis: an iron-dependent form of nonapoptotic cell death. Cell 149, 1060-1072.

[4] W.S. Yang, R. SriRamaratnam, M.E. Welsch, K. Shimada, R. Skouta, V.S. Viswanathan, J.H. Cheah, P.A. Clemons, A.F. Shamji, C.B. Clish, L.M. Brown, A.W. Girotti, V.W. Cornish, S.L. Schreiber, B.R. Stockwell, Regulation of ferroptotic cancer death by GPX4. Cell 156 (2014) 317-331.

[5] J. Lewerenz, S.J. Hewett, Y. Huang, M. Lambros, P.W. Gout, P.W. Kalivas, A. Massie, I. Smolders, A. Methner, M. Pergande, S.B. Smith, V. Ganapathy, P. Maher, The cystine/glutamate antiporter system xc- in health and disease: from molecular mechanisms to novel therapeutic opportunities. Antioxid. Redox Signal. 18 (2013) 522-555.

[6] P. Koppula L. Zhuang B. Gan, Cystine transporter SLC7A11/xCT in cancer: ferroptosis, nutrient dependency, and cancer therapy, Protein Cell (2020) doi.org/10.1007/s13238-020-00789-5.

[7] S.J. Dixon, B.R. Stockwell, The hallmarks of ferroptosis, Annu. Rev. Cancer Biol. 3 (2019) 35-54.

[8] C.S. Shin, P. Mishra, J.D. Watrous, V. Carelli, M. D’Aurelio, M. Jain, D.C. Chan, The glutamate/cystine xCT antiporter antagonaizes glutamine metabolism and reduces nutrient flexibility, Nat. Commun. 8 (2017) 15074.

[9] P. Koppula, Y. Zhang, J. Shi, W. Li, B. Gan, The glutamate/cystine antiporter SLC7A11/xCT enhances cancer cell dependency on glucose by exporting glutamate, J. Biol. Chem. 292 (2017) 14240-14249. 
[10] T. Goji, K. Takahara, M. Negishi, H. Katoh, Cystine uptake through the cystine/glutamine antiporter $\mathrm{xCT}$ triggers glioblastoma cell death under glucose deprivation, J. Biol. Chem. 292 (2017) 19721-19732.

[11] B. Hassannia, P. Vandenabeele, T.V. Berghe, Targeting ferroptosis to iron out cancer, Cancer Cell 35 (2019) 830-849.

[12] J. Liu, F. Kuang, G. Kroemer, D.J. Klionsky, D. Kang, D. Tang, Autophagy-dependent ferroptosis: machinery and regulation, Cell Chem. Biol. 27 (2020) 420-435.

[13] W.S. Yang, B.R. Sockwell, Synthetic lethal screening identifies compounds activating iron-dependent nonapoptotic cell death in oncogenic Ras harboring cancer cells, Chem. Biol. 15 (2008) 234-245.

[14] M. Gao, P. Monian, N. Quadri, R. Ramasamy, X. Jiang, Glutaminolysis and transferrin regulate ferroptosis, Mol. Cell 59 (2015) 298-308.

[15] W. Hou, Y. Xie, X. Song, M.T. Lotze, H.J. Zeh III, R. Kang, D. Tang, Autophagy promotes ferroptosis by degradation of ferritin, Autophagy 12 (2016) 1425-1428.

[16] M. Gao, P. Monian, Q. Pan, W. Zhang, J. Xiang, X. Jiang, Ferroptosis is an autophagic cell death process, Cell Res 26 (2016) 1021-1032.

[17] J. Du, T. Wang, Y. Li, Y. Zhou, X. Wang, X. Yu, X. Ren, Y. An, Y. Wu, W. Sun, W. Fan, Q. Zhu, Y. Wang, X. Tong, DHA inhibits proliferation and induces ferroptosis of leukemia cells through autophagy dependent degradation of ferritin, Free Radic. Biol. Med. 131 (2019) 356-369.

[18] E. Park, S.W. Chung, ROS-mediated autophagy increases intracellular iron levels and ferroptosis by ferritin and transferrin receptor regulation, Cell Death Dis. 10 (2019) 822.

[19] N. Geng, B.J. Shi, S.L. Li, Z.Y. Zhong, Y.C. Li, W.L. Xua, H. Zhou, J.H. Cai, Knockdown of ferroportin accelerates erastin-induced ferroptosis in neuroblastoma cells, Eur. Rev. Med. Pharmacol. Sci. 22 (2018) 38263836.

[20] C.W. Brown, J.J. Amante, P. Chhoy, A.L. Elaimy, H. Liu, L.J. Zhu, C.E. Baer, S.J. Dixon, AM. Mercurio, Prominin2 drives ferroptosis resistance by stimulating iron export, Dev. Cell 51 (2019) 575-586. 
[21] M.Y. Kwon, E. Park, S.J. Lee, S.W. Chung, Heme oxygenase-1 accelerates erastin-induced ferroptotic cell death, Oncotarget 6 (2015) 24393-24403.

[22] L.C. Chang, S.K. Chiang, S.E. Chen, Y.L. Yu, R.H. Chou, W.C. Chang, Heme oxygenase-1 mediates BAY 11-7085 induced ferroptosis, Cancer Lett. 416 (2018) 124-137.

[23] Y. Katoh, S. Michisaka, S. Nozaki, T. Funabashi, T. Hirano, R. Takei, K. Nakayama, Practical method for targeted disruption of cilia-related genes by using CRISPR/Cas9-mediated homology-independent knock-in system, Mol. Biol. Cell 28 (2017) 898-906.

[24] J.M. Goodwin, W.E. Dowdle, R. DeJesus, Z. Wang, P. Bergman, M. Kobylarz, A. Lindeman, R.J. Xavier, G. McAllister, B. Nyfeler, G. Hoffman, L.O. Murphy, Autophagy-independent lysosomal targeting regulated by ULK1/2-FIP200 and ATG9. Cell Rep 20 (2017) 2341-2356.

[25] J.D. Mancias, X. Wang, S.P. Gygi, J.W. Harper, A.C. Kimmelman, Quantitative proteomics identifies NCOA4 as the cargo receptor mediating ferritinophagy. Nature 509 (2014) 105-109.

[26] N. Santana-Codina, J.D. Mancias, The role of NCOA4-mediated ferritinophagy in health and disease, Pharmaceuticals 11(2018) 114.

[27] J. Liu, F. Kuang, G. Kroemer, D.J. Klionsky, R. Kang, D. Tang, Autophagy-dependent ferroptosis: machinery and regulation, Cell Chem. Biol. 27 (2020) 420-435.

[28] Y. Kabeya, N. Mizushima, T. Ueno, A. Yamamoto, T. Kirisako, T. Noda, E. Kominami, Y. Ohsumi, T. Yoshimori, LC3, a mammalian homologue of yeast Apg8p, is localized in autophagosome membranes after processing, EMBO J 19 (2000) 5720-5728.

[29] N. Mizushima, T. Yoshimori, How to interpret LC3 immunoblotting, Autophagy 3 (2007) 542-545.

[30] T. Imai, S. Iwata, T. Hirayama, H. Nagasawa, S. Nakamura, M. Shimazawa, H. Hara, Intracellular $\mathrm{Fe}^{2+}$ accumulation in endothelial cells and pericytes induces blood-brain barrier dysfunction in secondary brain injury after brain hemorrhage, Sci. Rep. 9 (2019) 6228. 
[31] M. Gao, J. Yi, J. Zhu, A.M. Minikes, P. Monian, C.B. Thompson, X. Jiang, Role of mitochondria in ferroptosis, Mol. Cell 73 (2019) 354-363.

[32] T.Z. Kidane, E. Sauble, M.C. Linder, Release of iron from ferritin requires lysosomal activity, Am. J. Physiol. Cell Physiol. 291 (2006) C445-C455.

[33] Y. Zhang, M. Mikhael, D. Xu, Y. Li, S. Soe-Lin, B. Ning, W. Li, G. Nie, Y. Zhao, P. Ponka, Lysosomal proteolysis is the primary degradation pathway for cytosolic ferritin and cytosolic ferritin degradation is necessary for iron exit, Antioxid. Redox Signal. 13 (2010) 999-1009. 


\section{Figure legends}

Figure 1. Cystine deprivation, but not GSH depletion, induces ferroptosis in glioblastoma cells.

A. T98G and A172 cells were placed in medium with or without cystine $(0.2 \mathrm{mM})$ and BSO $(100 \mu \mathrm{M})$ for $24 \mathrm{~h}$. Cell death was quantified using an LDH release assay. Cells treated with $0.1 \%$ Tween 20 were used to calculate $100 \%$ cell death.

B. T98G and A172 cells were placed in medium with or without cystine and BSO for $15 \mathrm{~h}$. Cellular GSH was measured and the GSH level relative to that of control (+cystine, $-\mathrm{BSO})$ is shown.

C. T98G and A172 cells were placed in medium with or without cystine, DFO $(100 \mu \mathrm{M})$, and Fer-1 $(1 \mu \mathrm{M})$ for $24 \mathrm{~h}$. Error bars represent S.D. $(\mathrm{n}=3) . *^{* *}, p<0.001$, calculated by one-way ANOVA with Tukey's post hoc test.

D. Immunoblotting analysis of $\mathrm{T} 98 \mathrm{G}$ and $\mathrm{A} 172$ cells cultured in medium with or without cystine $(0.2 \mathrm{mM})$ for $15 \mathrm{~h}$.

E. Immunoblotting analysis of $\mathrm{T} 98 \mathrm{G}$ and A172 cells cultured in 
medium with or without BSO for $15 \mathrm{~h}$.

Fig. 2. Ferritinophagy plays an important role in cystine deprivationinduced cell death.

A. Immunoblotting analysis of T98G and A172 cells cultured in medium with or without cystine $(0.2 \mathrm{mM})$ and BafA1 $(1 \mu \mathrm{M})$ for $15 \mathrm{~h}$.

B. T98G and A172 cells were placed in medium with or without cystine and BafA1 for $24 \mathrm{~h}$. Cell death was quantified using an LDH release assay. Cells treated with $0.1 \%$ Tween 20 were used to calculate $100 \%$ cell death.

C. Immunoblotting analysis of control (Control KO) and NCOA4deficient (NCOA4 KO) T98G cells.

D. Immunoblotting analysis of control and NCOA4-deficient T98G cells cultured in medium with or without cystine $(0.2 \mathrm{mM})$ for $15 \mathrm{~h}$.

E. Control and NCOA4-deficient T98G cells were placed in medium with or without cystine for $24 \mathrm{~h}$. Error bars represent S.D. $(\mathrm{n}=3)$. ${ }^{* * *}, p<$ 
0.001, calculated by one-way ANOVA with Tukey's post hoc test.

F. Immunoblotting analysis of T98G and A172 cells cultured in medium with or without cystine $(0.2 \mathrm{mM})$ and BafA1 $(1 \mu \mathrm{M})$ for $15 \mathrm{~h}$.

Fig. 3. Intracellular iron accumulation induces cell death in BSO-treated cells.

A. T98G and A172 cells were placed in medium with or without BSO $(100 \mu \mathrm{M})$ and FAS $(300 \mu \mathrm{M})$ for $24 \mathrm{~h}$.

B. T98G and A172 cells were placed in medium with or without BSO and hemin $(10 \mu \mathrm{M})$ for $24 \mathrm{~h}$.

C. T98G and A172 cells were placed in medium with or without cystine, FAS $(300 \mu \mathrm{M})$, and BafA1 for $24 \mathrm{~h}$.

D. T98G and A172 cells were placed in medium with or without cystine, hemin $(10 \mu \mathrm{M})$, and BafA1 for $24 \mathrm{~h}$. Cell death was quantified using an $\mathrm{LDH}$ release assay. Cells treated with $0.1 \%$ Tween 20 were used to calculate $100 \%$ cell death. Error bars represent S.D. $(\mathrm{n}=3) . * * *, p<0.001$; 
**, $p<0.01$, calculated by one-way ANOVA with Tukey's post hoc test.

Fig. 4. Glutamine is required for cystine deprivation-induced degradation of FTH1 and cell death.

A. T98G and A172 cells were placed in medium with or without cystine $(0.2 \mathrm{mM})$ and glutamine $(\mathrm{Gln}, 2 \mathrm{mM})$ for $36 \mathrm{~h}$.

B. T98G and A172 cells were placed in medium with or without cystine, glutamine, and FAS $(300 \mu \mathrm{M})$ for $24 \mathrm{~h}$. Cell death was quantified using an LDH release assay. Cells treated with $0.1 \%$ Tween 20 were used to calculate $100 \%$ cell death.

C. Immunoblotting analysis of T98G and A172 cells cultured in medium with or without cystine and glutamine for $15 \mathrm{~h}$.

D. T98G and A172 cells were placed in medium with or without cystine and glutamine for $15 \mathrm{~h}$. Cellular GSH was measured and the GSH level relative to that of control (+cystine, +glutamine) is shown. Error bars represent S.D. $(\mathrm{n}=3)$. ***, $p<0.001$, calculated by one-way ANOVA with 
Tukey's post hoc test. 
A

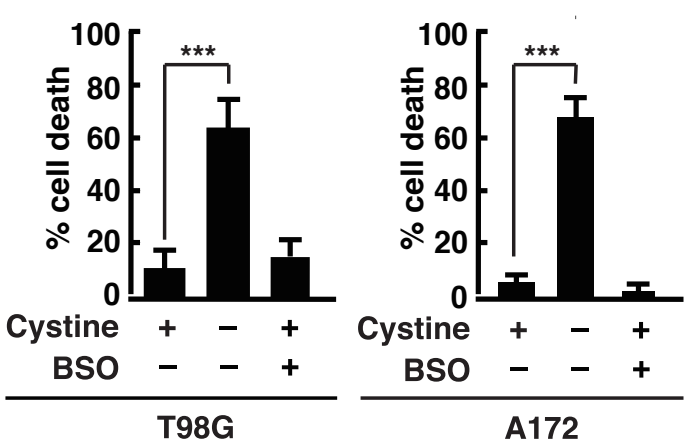

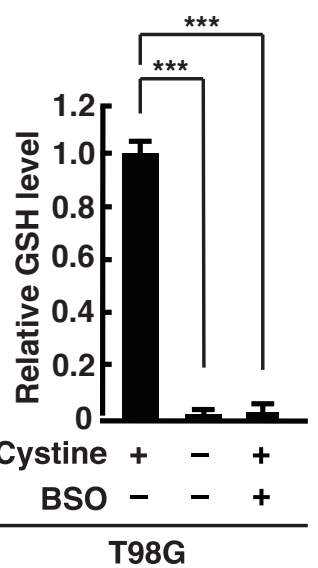

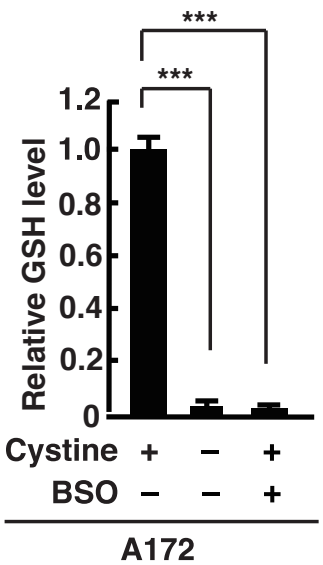

C
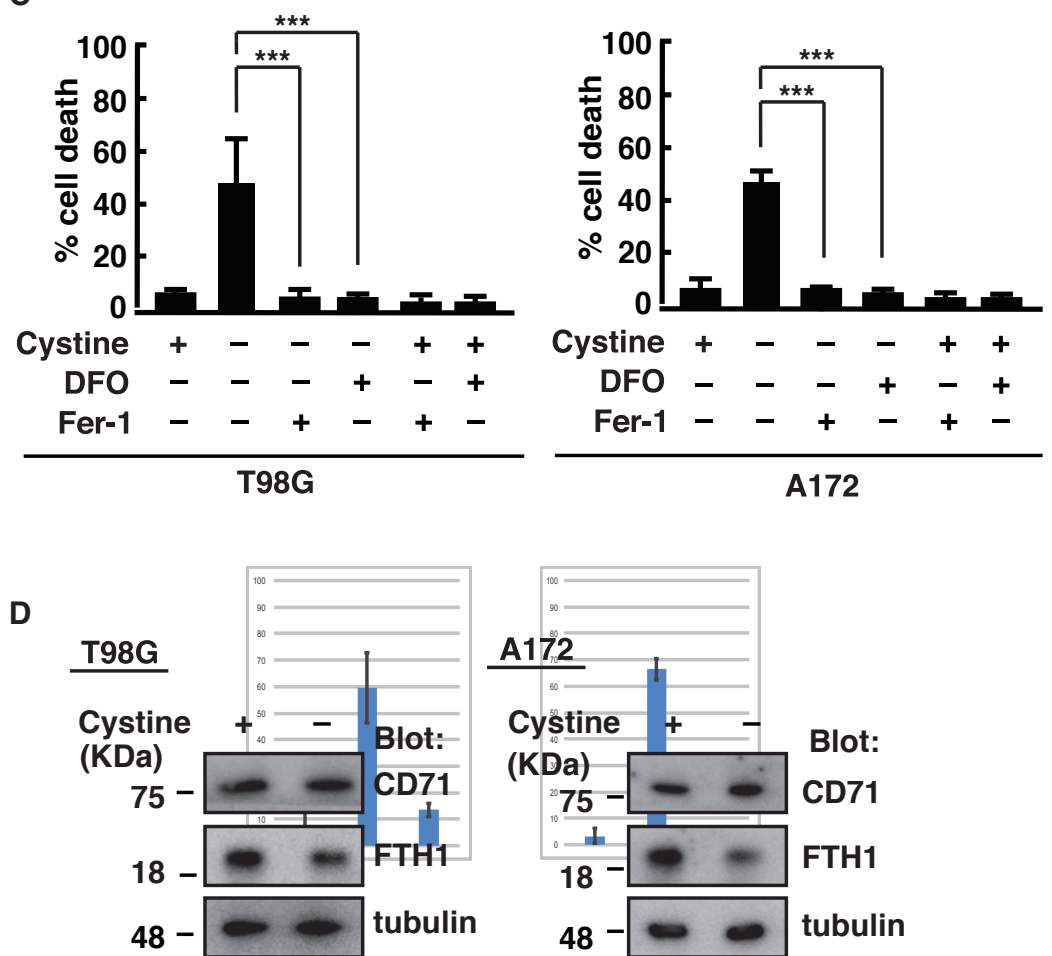

E

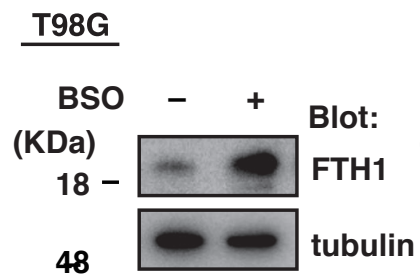

$\underline{A 172}$

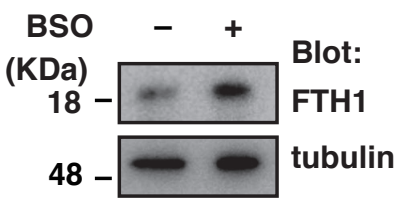

Figure 1 
A T98G

A172

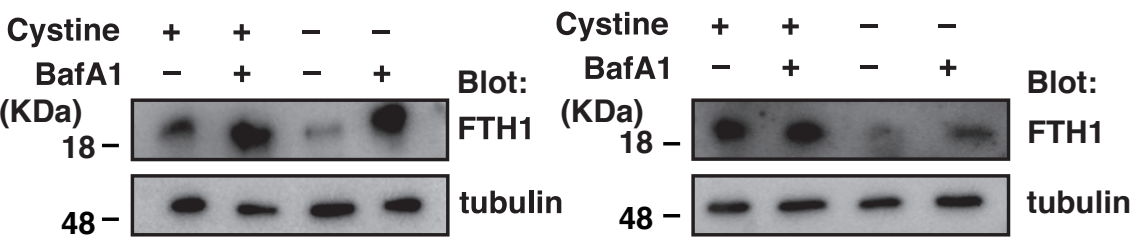

B

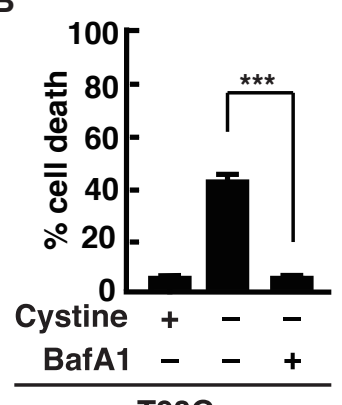

T98G

C

F

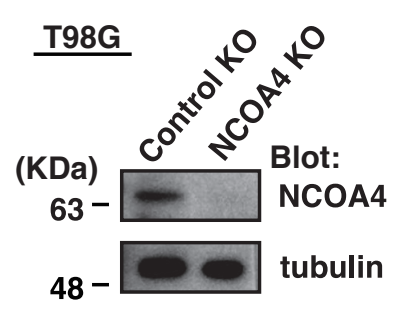

T98G

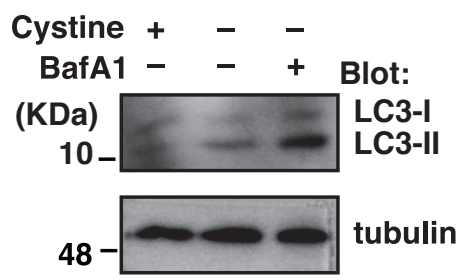

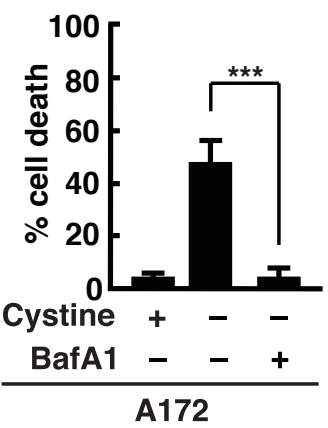

D T98G

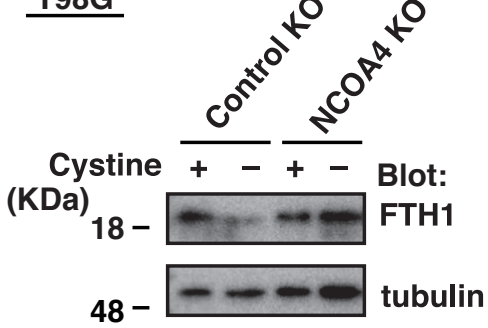

A172

Cystine + - -

BafA1 - - + Blot:

(KDa) LC3-I

10- 4C3-II

$48-\square$ tubulin
E

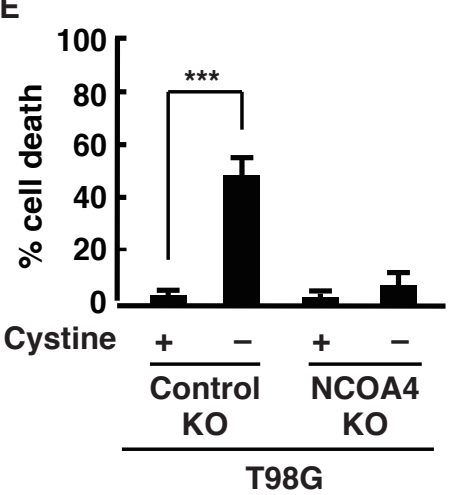

Figure 2 


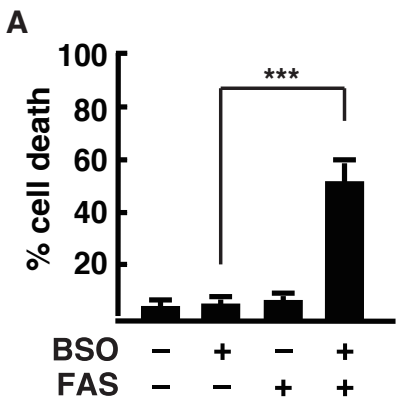

T98G
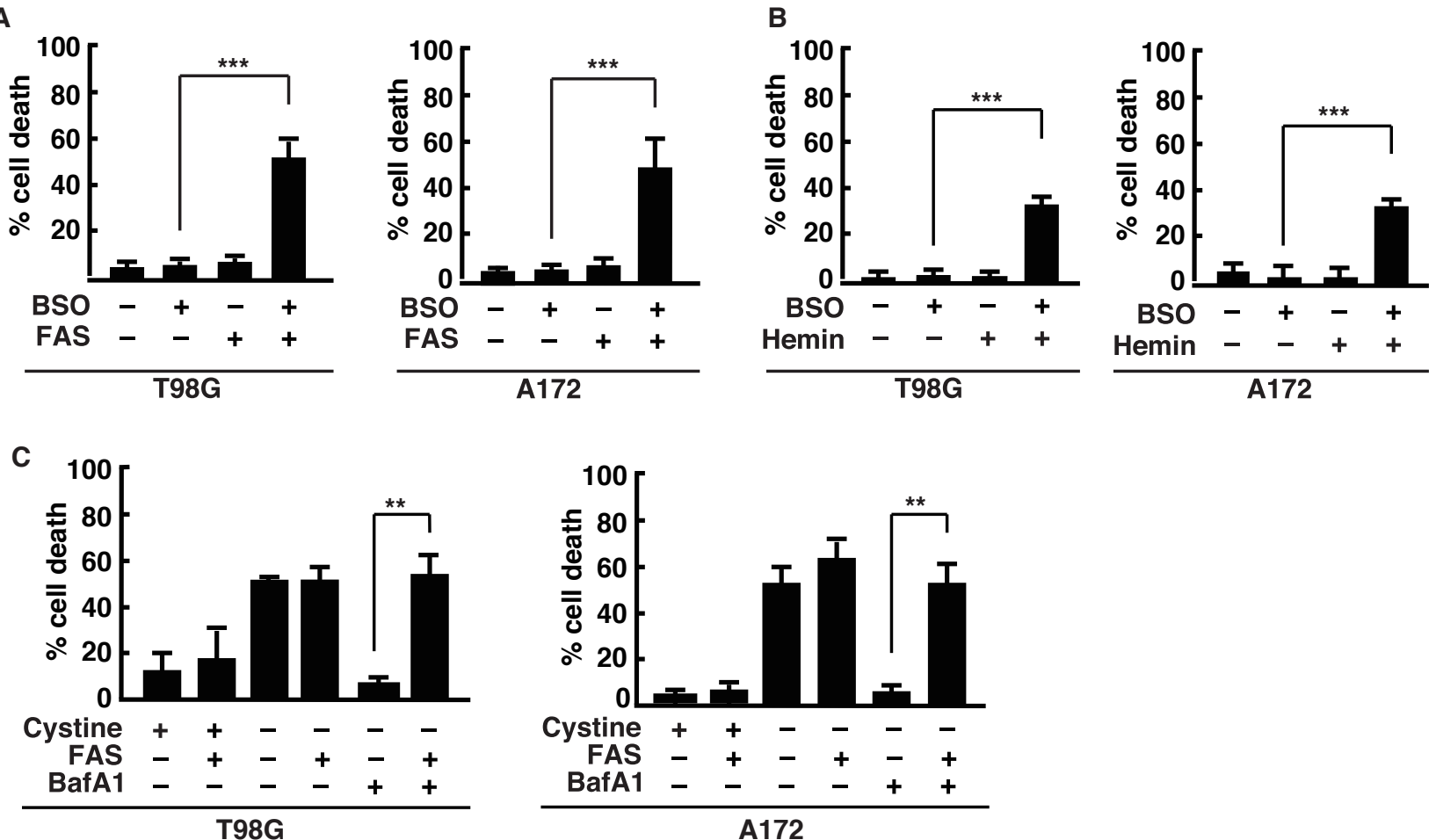

D
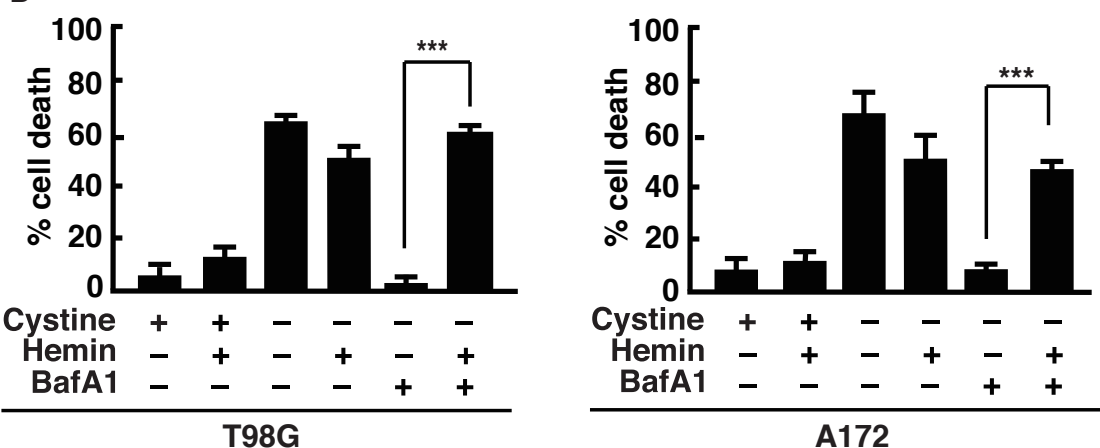

Figure 3 


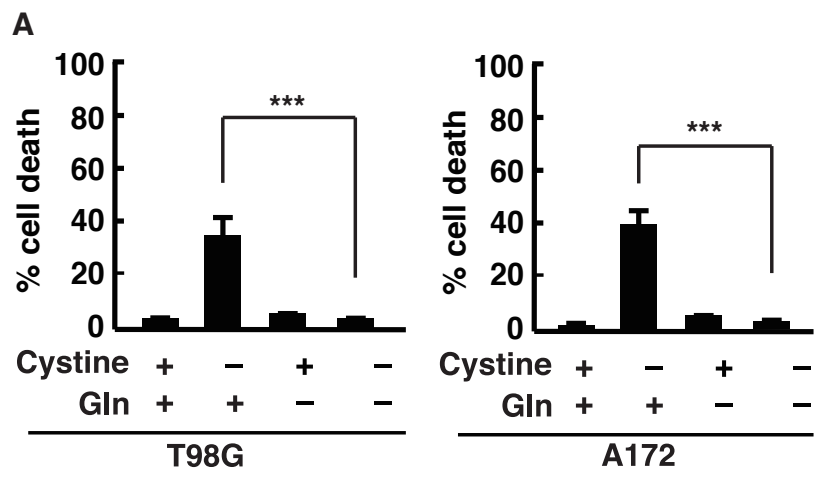

B
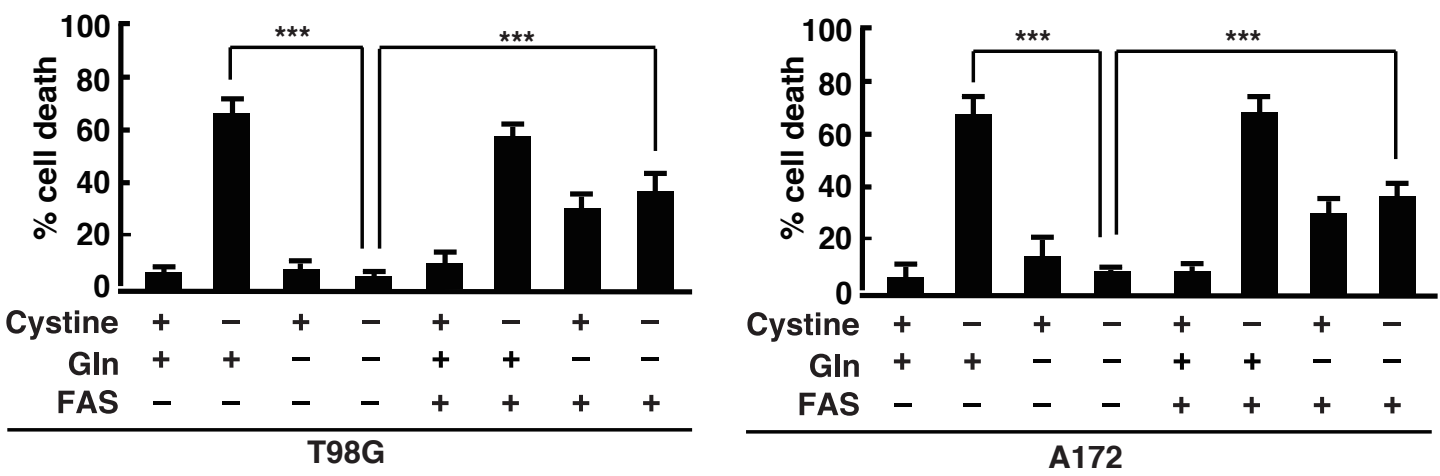

C

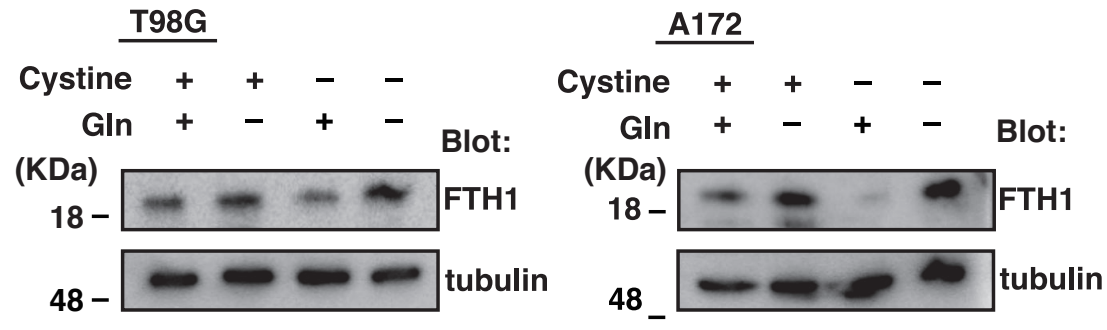

D
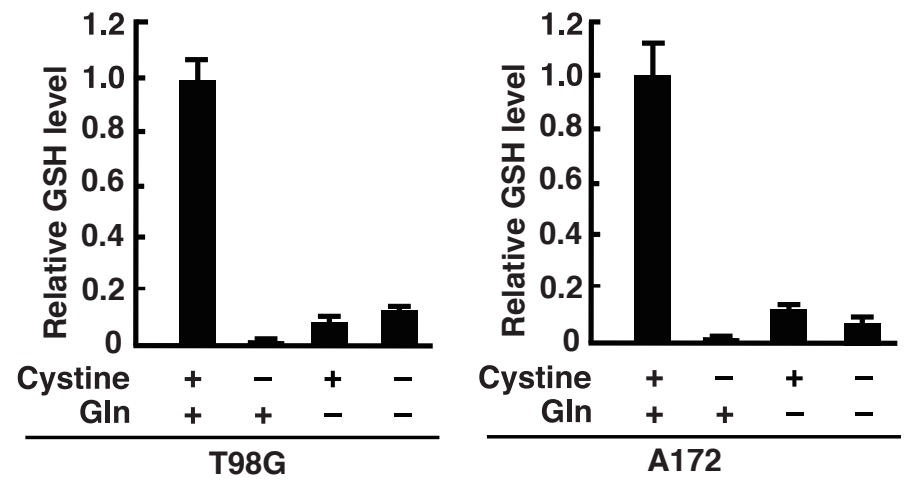

Figure 4 